\title{
Introducing the Patterson Cylindrical Projection
}

Tom Patterson

US National Park Service tom_patterson@nps.gov
Bojan Šavrič

Oregon State University savricb@geo.oregonstate.edu
Bernhard Jenny

Oregon State University

jennyb@geo.oregonstate.edu

The Patterson cylindrical, a new projection designed for general-purpose mapmaking, is an alternative to other cylindrical projections. It is positioned between the Plate Carrée projection, which has a 1:2 aspect ratio, and the Miller 1 projection, which excessively exaggerates the size of polar areas. The Patterson cylindrical balances polar exaggeration against maintaining the familiar shape of continents and has a compact height-to-width aspect ratio. Creating the projection started with a graphical template made in Flex Projector that served as a guide for developing the polynomial equations, which are introduced in this article. The reference source code is available in the Java Map Projection Library.
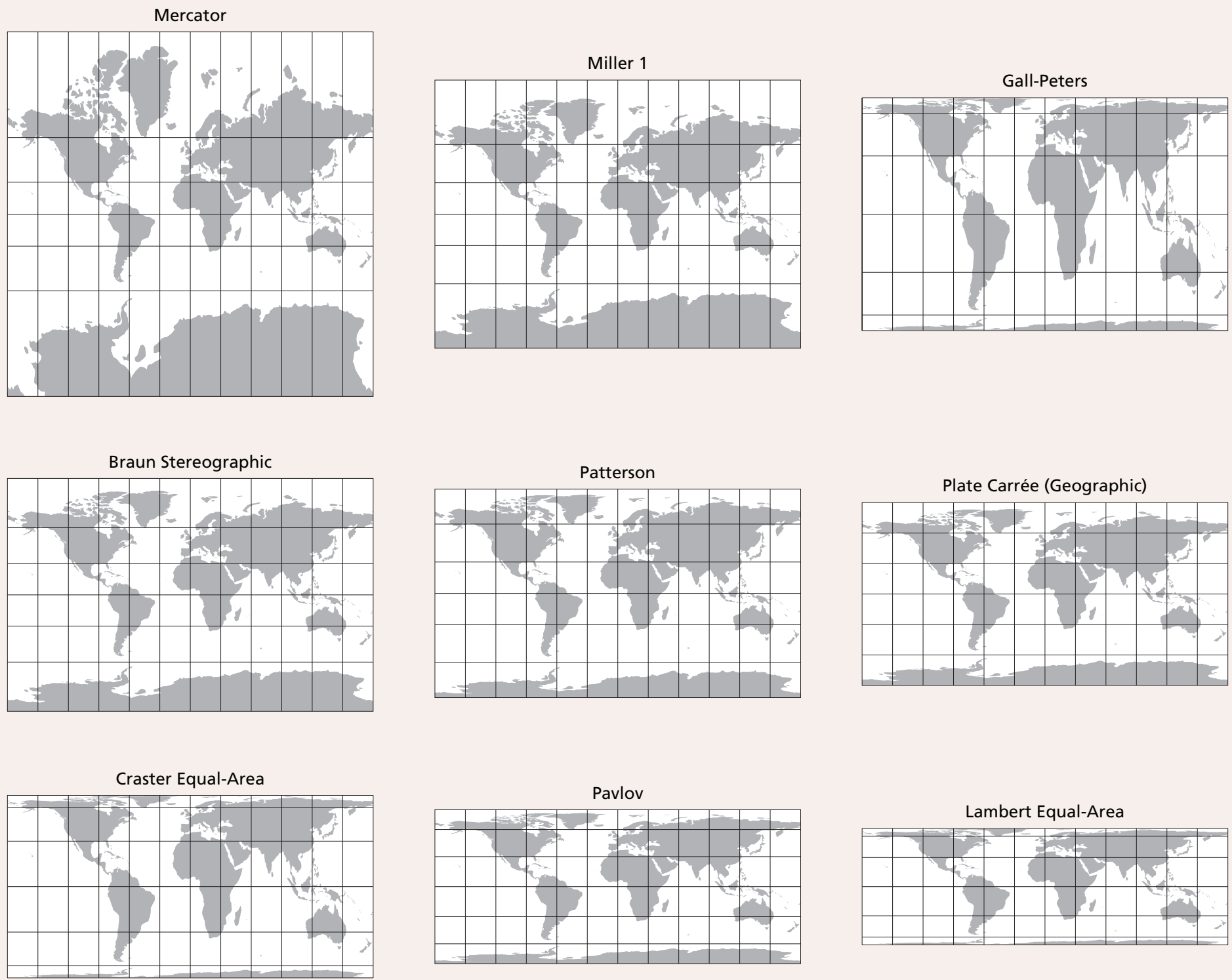

Figure 1. The Patterson (middle) compared to other commonly used cylindrical projections. 


\section{DESIGN OF THE PATTERSON CYLINDRICAL PROJECTION}

Cylindrical map projections depict the nearly spherical Earth as a rectangle, showing meridians and parallels as straight lines. The North and South Poles, actually points on the Earth, appear as lines across the top and bottom of the map. Designing a cylindrical projection therefore requires finding, for higher latitude areas, a compromise between two extremes: maintaining the general shape of map features and exaggerating their relative area, or vertically compressing map features and maintaining their area. One extreme is the conformal Mercator projection, developed for navigation during the age of sail, which grossly exaggerates high-latitude areas; the other extreme is the family of equal area cylindrical projections, such as the Gall-Peters, Craster, or Lambert cylindrical, which come at the expense of vertically compressed and deformed shapes (Figure 1). The Patterson projection is an addition to a variety of existing compromise cylindrical projections (Jenny et al. 2015), but has its own unique traits. The Patterson packages the world in a relatively compact rectangle with a height-to-width aspect ratio of about 0.57 . The exaggeration of high-latitude areas is considerably lessened compared to the Miller 1 and other similar projections.

The Patterson projection derives from the Miller 1 projection, modified graphically using Flex Projector software (Jenny et al. 2008; 2010). The Miller 1 projection, introduced by Osborn Maitland Miller (Miller 1942; Monmonier 2002), was a good starting point because it depicts features in tropical and mid-latitude regions with relatively balanced proportions. For example, the tapered
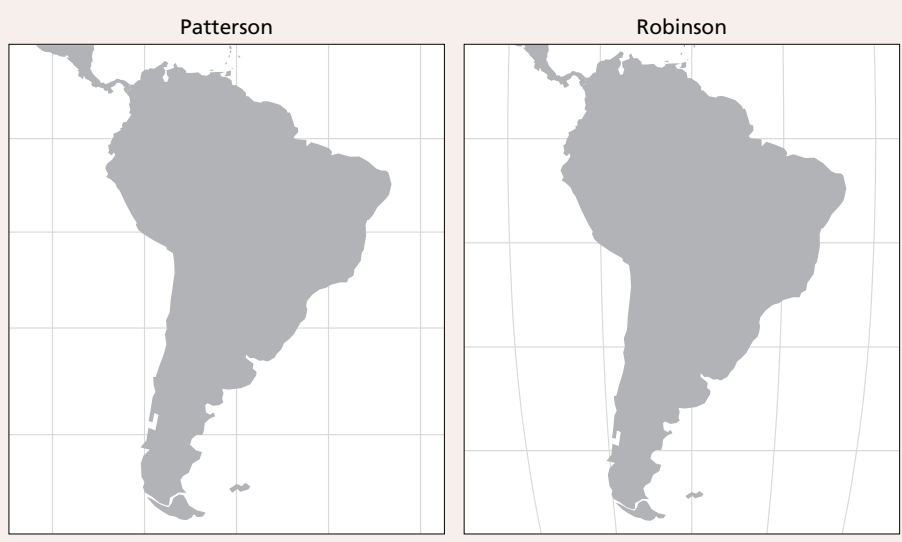

Figure 2. Excerpts of world maps with the same width centered on 0 north, 60 west. The proportions of South America on the Patterson projection are similar to that of the Robinson, a pseudocylindrical projection.

shape of South America does not look too stretched or stubby and compares favorably to the popular Robinson projection (Figure 2). The Miller 1 and Patterson projections are nearly identical between 55 degrees north and south latitude, where most of the people on Earth live.

Developing the Patterson cylindrical projection involved three steps. In the first step, we modified the Miller 1 projection, which resulted in the Compact Miller projection. In the second step, we compressed higher latitudes, which resulted in a graphical template for the Patterson projection. In the third step, we developed the projection equations.

\section{STEP 1: DESIGNING THE COMPACT MILLER PROJECTION}

Unlike the Miller I, which has increasing spacing between lines of latitude moving from the equator toward the poles, we first applied constant spacing to high-latitude regions. Beyond 55 degrees, the spacing between lines of latitude remains constant, as on an equirectangular projection. This adjustment yielded the intermediate Compact Miller projection, which compresses polar areas in comparison to the Miller 1 projection (Figure 3). The design considerations for the development of the Compact Miller projection and its equations are described by Jenny et al. (2015).
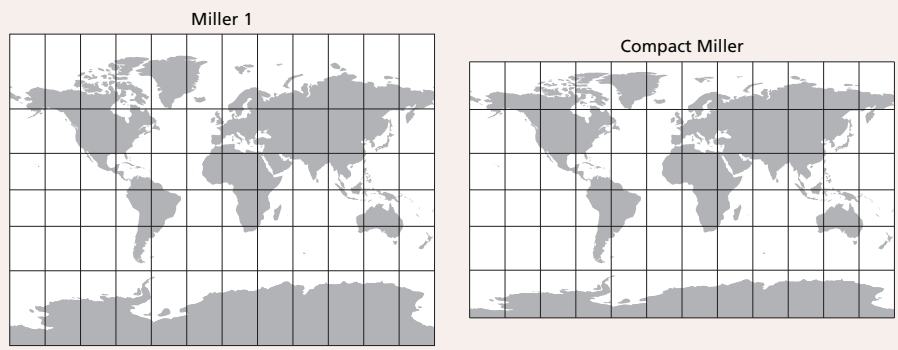

Figure 3. The Miller 1 and Compact Miller, an intermediate production step, are identical from latitude 55 north to 55 south. 


\section{STEP 2: DESIGNING THE PATTERSON PROJECTION}

Although the Compact Miller greatly reduces the area of polar regions compared to the original Miller 1 , Antarctica and the high arctic still appear disproportionately large. For example, Greenland, actually roughly equal in area to the Indian subcontinent, appears excessively large in the Compact Miller projection. A partial solution to this problem was to reduce the distance between lines of latitude poleward of 65 degrees. In Flex Projector, we applied the most compression to areas immediately adjacent to the poles. The compression gradually diminishes, and ends at about 65 degrees latitude. This adjustment created a graphical template of the Patterson cylindrical projection for which the final equations were developed (Figure 4).
One concern when applying polar compression was to maintain the distinctive shape of Alaska and other landmasses at high latitudes. Figure 5 shows the relative distance between lines of latitude for the Patterson projection, which steadily increase in length moving from the equator to 55 degrees, level off around 60 degrees, and then decrease toward the poles. This pattern is a distinguishing trait of the Patterson projection, as all other commonly used cylindrical projections do not vary latitudinal distances in this manner. On the Patterson projection, the polar compression is only obvious when a graticule with latitude increments of 15 degrees or less is used.
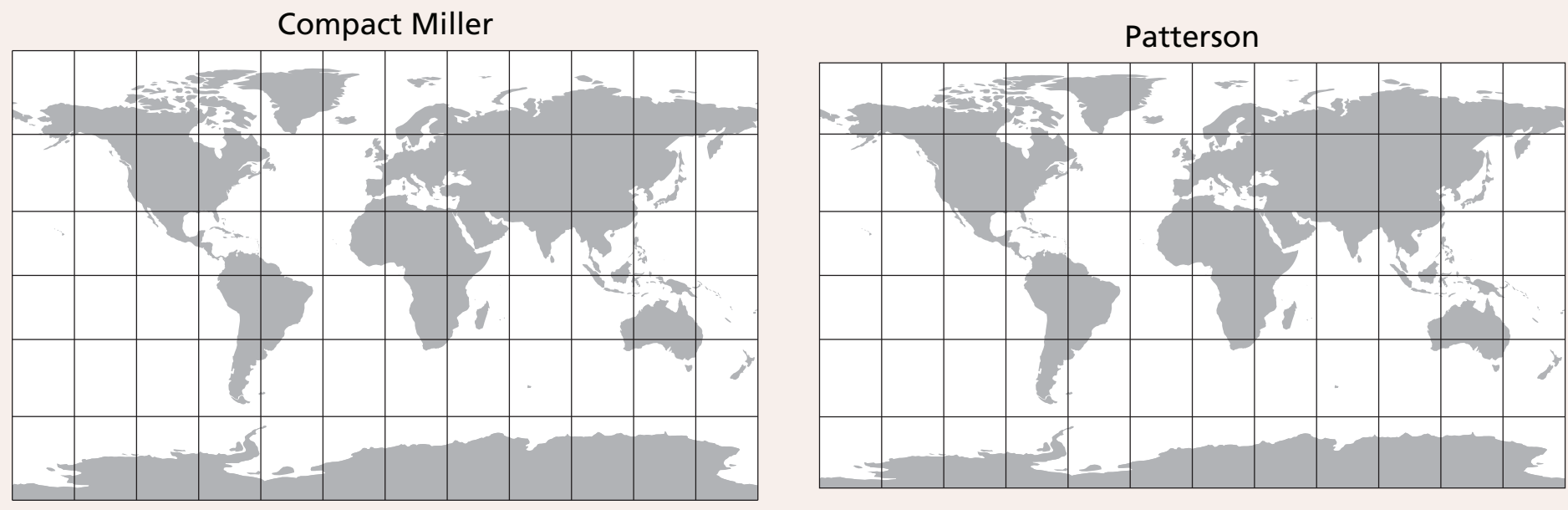

Figure 4. Compared to the Compact Miller, the Patterson has compressed polar regions.
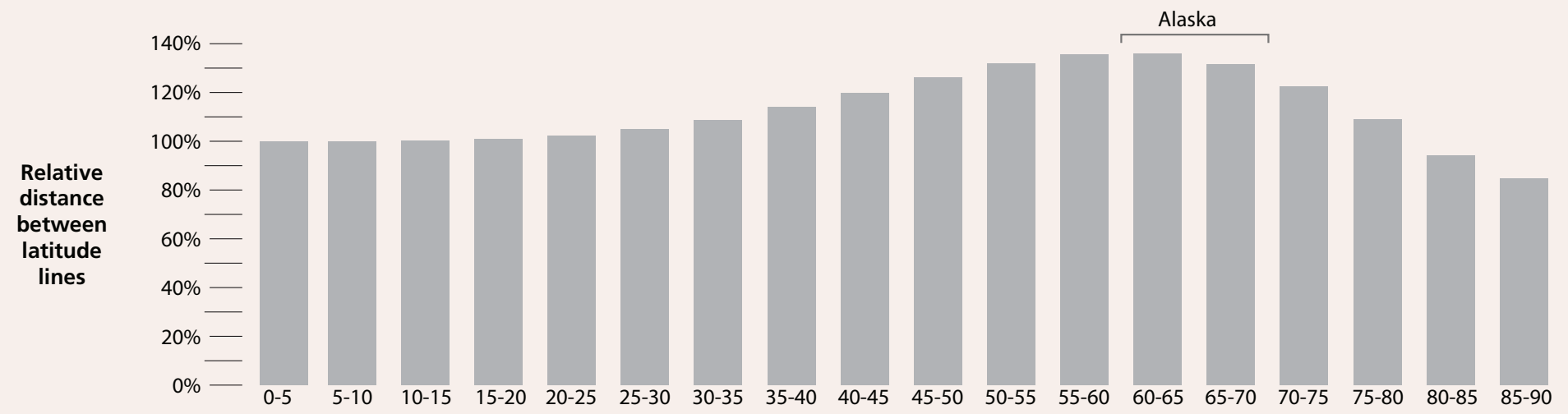

Degrees latitude

Figure 5. Relative distance between latitude lines on the Patterson projection. 


\section{STEP 3: FINDING EQUATIONS FOR THE PATTERSON PROJECTION}

THE LAST STEP involved developing polynomial equations approximating the Flex Projector template for the Patterson projection. The process was similar to that used for the Natural Earth projection (Šavrič et al. 2011). Between 55 degrees north and south latitude, the polynomial equation does not exactly match the Miller 1 , but the differences are only noticeable at high magnification near 15 degrees latitude. Equation 1 transforms spherical longitude and latitude coordinates to projected coordinates.

$$
\begin{gathered}
x=\lambda \\
y=c_{1} \cdot \varphi+c_{2} \cdot \varphi^{5}+c_{3} \cdot \varphi^{7}+c_{4} \cdot \varphi^{9}
\end{gathered}
$$

In Equation 1, $x$ and $y$ are the projected coordinates, $\varphi$ and $\lambda$ are the latitude and longitude, and the polynomial coefficients are $c_{1}=1.0148, c_{2}=0.23185, c_{3}=-0.14499$, and $c_{4}=$ 0.02406 . The computational cost per point consists of six multiplications and three additions when the polynomial in Equation 1 is factorized as in Equation 2:

$$
y=\varphi \cdot\left(c_{1}+\varphi^{2} \cdot \varphi^{2} \cdot\left(c_{2}+\varphi^{2} \cdot\left(c_{3}+\varphi^{2} \cdot c_{4}\right)\right)\right)
$$

(Equation 2)

To convert projected coordinates to spherical coordinates, the Newton-Raphson method is used to find the latitude $\varphi$ from the $y$ equation; longitude $\lambda$ is computed by inverting the $x$ equation.

\section{AVAILABILITY}

The Patterson cylindrical projection is currently available in the latest version of Flex Projector (available for free at flexprojector.com). With Flex Projector, you can make maps from vector and raster geospatial data (Figure 6). If the Patterson projection is not yet offered in your favorite cartographic or GIS software, please direct the developer to the Java Map Projection Library (github. com/OSUCartography/JMapProjLib) to obtain the reference source code.

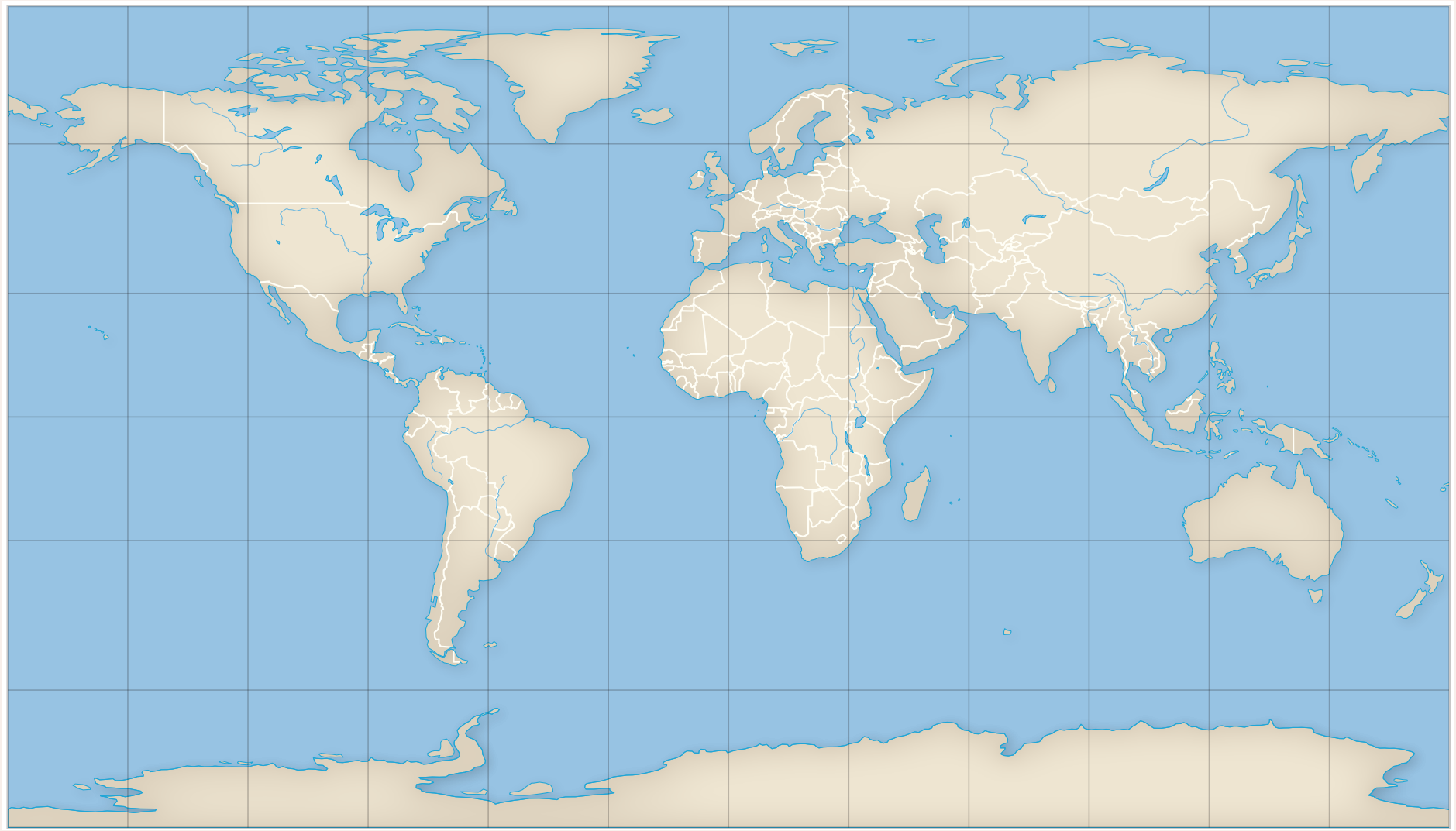

Figure 6. Map with the Patterson projection created with Flex Projector using Natural Earth $110 \mathrm{~m}$ vector data. 
Jenny, B., T. Patterson, and L. Hurni. 2008. "Flex

Projector-Interactive software for designing world map projections." Cartographic Perspectives 59: 12-27. doi: $10.14714 /$ CP59.245.

Jenny, B., T. Patterson, and L. Hurni. 2010. "Graphical design of world map projections." International Journal of Geographical Information Science 24 (11): 1687-1702. doi: $10.1080 / 13658811003596101$.

Jenny, B., B. Šavrič, and T. Patterson. 2015. "A compromise aspect-adaptive cylindrical projection for world maps." International Journal of Geographical Information Science. Forthcoming. doi: 10.1080/13658816.2014.997734.
Miller, O. M. 1942. "Notes on cylindrical world map projections." Geographical Review: 424-430. doi: $10.2307 / 210384$.

Monmonier, M. 2002. "All over the map: Miller time: Modifying Mercator.” Mercator's World 7 (6): 52-54.

Šavrič, B., B. Jenny, T. Patterson, D. Petrovič, and L. Hurni. 2011. "A polynomial equation for the Natural Earth projection." Cartography and Geographic Information Science 38 (4): 363-372. doi: $10.1559 / 15230406384363$. 